\title{
Remarks on John Cahn's Career
}

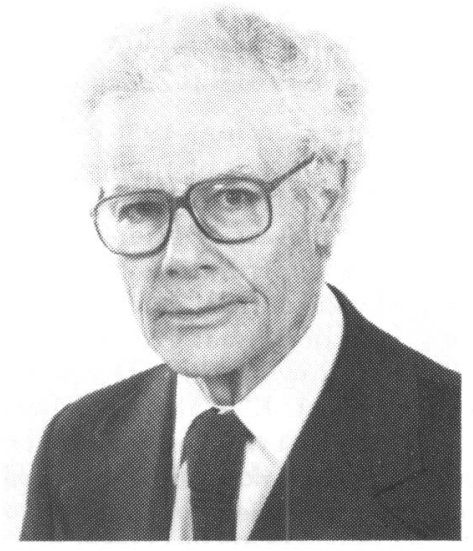

The research, instruction, and critiques of John Cahn have constituted a powerful driving force for the advancement of materials science over the past three decades. They have vitally affected every area of the field and have strongly influenced statistical physics as well. It seems safe to say that without them, our knowledge and understanding of materials science would have been, today, in a far more primitive and disjointed state.

Cahn obtained his $\mathrm{PhD}$ in physical chemistry at Berkeley in a milieu where there was a strong emphasis on classical thermodynamics. Judging from his later career, this emphasis apparently suited his natural inclinations and interests very well. Following Berkeley, he spent two years at Chicago's Institute for the Study of Metals during the exciting period of Cyril Smith's directorship. Undoubtedly the ambience at the Institute arising from the pioneering metallurgical researches of $S$ mith, Clarence Zener, Charles Barrett, and their colleagues played an important part in attracting Cahn to materials science and its challenges.

In 1954 Cahn joined the Hollomon Metallurgy and Ceramics group of the G.E. Research Laboratory as a member of the Chemical Metallurgy section which I, at that time, headed. He immediately became interested in interfaces and their role in phase transformations and derived the conditions under which solid-state transformation kinetics should be dominated by nucleation at the boundaries, edges, or corners of grains. Later he developed the quantitative theory for preferential nucleation of incoherent phases on dislocations.

At this time, there was much confusion about the conditions for the transition from the nucleation and growth to the spinodal mode of phase separation. In retrospect, as Cahn pointed out in a review, this confusion might not have arisen had the early papers of Gibbs and van der Waals been better known and understood. Partly stimulated by Hillert's theory for the onedimensional development of composition modulations, Cahn and John Hilliard at- tacked the spinodal problem. Deeply inspired by the rigor and power of Gibbs' treatment of thermodynamics, they began by reading together Gibbs' paper on heterogeneous equilibria, virtually sentence by sentence and assuring themselves that they understood every step of the development. There resulted their classic papers on the free-energy of non-uniform systems in which was derived the Cahn-Hilliard equation for this free energy, the conditions for the spinodal and for the nucleation-controlled modes of phase separation. The CahnHilliard equation has had great impact on materials science and is often the starting point of statistical physical treatments of a variety of interfacial phenomena.

Following these contributions, partly during the period 1960-1961 which he spent at Cambridge on a Guggenheim Fellowship, Cahn derived the theory for the effects of coherency strains on the thermodynamics of phase separation and on the occurrence and early course of spinodal precipitation. This theory explained for the first time the early morphologies of spinodal processes, including the beautifully periodic patterns exhibited by certain precipitate dispersions, e.g., in the Alnico alloys. Also derived from this theory was Cahn's equation for interdiffusion in the presence of extremely high concentration gradients. This equation has been confirmed by a number of experimental studies, perhaps most impressively by those of Hilliard and his students on interdiffusion in compositionally modulated films of very short wave length. Cahn's theory for the coherent spinodal also has greatly illuminated the understanding of the dependence of magnetic and mechanical properties on interphase morphology and has provided valuable guidance for alloy design.

In another important group of papers, some written at G.E. and others at MIT and NBS, Cahn analyzed the interplay, and the consequences, between impurity or heat transport and interfacial rearrangement in various interface migration processes. These analyses explained: (1) the incomplete

\section{David Turnbull}

depletion of solute in boundary-controlled cellular precipitation; (2) "impurity drag" in grain boundary migration (a similar theory for this effect was derived independently by Lücke); (3) the origin of "solute trapping" in rapid solidification processing - the thermodynamic requirements for such trapping were set forth by Cahn and his student, J. C. Baker at MIT; (4) the persistence of morphological stability in certain crystals growing at departures from equilibrium well beyond those predicted for instability onset by the Mullins-Sekerka theory.

More recently at NBS, in collaboration with Francis Larché and others, Cahn developed, in the spirit of Gibbs, a more rigorous and definitive treatment of the thermodynamics of stressed solids and applied it to the analysis of diffusional creep and other phenomena. Very recently Cahn collaborated with Dan Shechtman and others in the first recognition and characterization of a phase exhibiting icosahedral point group symmetry as well as a sharp diffraction pattern. The existence of such a form of matter was quite unexpected and has, as we all know, greatly excited the entire materials science community.

The foregoing listing of Cahn's contributions is far from complete, and scientists with interests differing from mine might compile quite different but equally impressive lists.

Apart from his direct contributions, Cahn has had a major influence on materials science through his critiques, private and public, of the studies and interpretations of others, e.g., of the DIGM phenomena. A generation of young materials scientists has beaten a path to his door for advice and instruction on their studies. It is, I think, fair to say that the prevalent standards of the field have been much elevated by these critiques.

Clearly John Cahn's record is one marked by towering achievements. It is indeed, most fitting that it be recognized by the Von Hippel Award.

David Turnbull, Harvard University 